\title{
Injection Locking in Distributed Feedback Semiconductor Lasers
}

\author{
Rongqing Hui, Alessandro D'Ottavi, Antonio Mecozzi, and Paolo Spano
}

\begin{abstract}
Injection locking properties of distributed feedback semiconductor lasers are studied systematically. Due to the high side mode suppression, these devices show different locking properties when compared to lasers having Fabry-Perot structures. The main result is the identification of four regimes for different injection levels. In particular, a symmetrical locking band at low optical injection level is confirmed. The presence of this symmetrical band can be exploited in some applications: as examples, the measurement of the linewidth enhancement factor $\alpha$ and the PSK modulation capability are reported.
\end{abstract}

\section{INTRODUCTION}

$\mathbf{I}_{\mathrm{n}}^{\mathrm{N}}$ NJECTION locking is a promising method to synchronize one (or several) free running oscillators to a master laser having, in general, a higher frequency purity. Injection locking of semiconductor lasers can be usefully employed in multichannel coherent optical transmission systems, and it is also a good method to investigate laser properties. Numerous studies on semiconductor lasers with external optical injection have been published so far [1]-[8]. However, most of the experimental measurements were done on Fabry-Perot (FP) laser diodes, where the presence of longitudinal modes having a comparable value of gain prevents an extensive investigation of the injection locking properties.

This paper reports, for the first time to the authors' knowledge, a systematic experimental investigation of the injection locking properties of distributed feedback (DFB) semiconductor lasers. In these lasers, the side longitudinal modes are highly suppressed by the distributed grating, so that injection locking can be maintained even in conditions in which the phase relation between the electric field present in the laser cavity and that injected from outside is such that the threshold gain is higher than that for the free running laser. These conditions cannot be achieved when using FP lasers due to the mode hopping among different longitudinal modes. Owing to this difference, the experimental results show a symmetric stable locking band in DFB lasers at low injection power, where FP lasers display a typical asymmetric band [4]. As a function of injection power, four different regimes have

Manuscript received October 18, 1990; revised February 11, 1991. This work was supported by the Fondazione Ugo Bordoni and the Italian P. T Administration.

The authors are with the Fondazione Ugo Bordoni, 00142, Rome, Italy. IEEE Log Number 9100604. been experimentally distinguished: 1) a symmetrical stable locking band; 2) two stable locking bands for positive and negative values of frequency detuning separated by an unstable region; 3) stable locking only for negative values of detuning; and 4) a bistable region. The measurements were performed by varying for more than 30 $\mathrm{dB}$ the injected optical power level. To explain the experimental results, a theory showing the importance of the nonlinear gain saturation term in stabilizing the injection locking has been developed.

We also propose a new method for measuring the linewidth enhancement factor $\alpha$ in DFB lasers based on the presence of the symmetric stable locking band at low injection level. This technique is simple and accurate because it does not require the knowledge of the value of the injected optical power, while similar methods previously proposed do require such knowledge [8].

Moreover, the symmetric stable locking band allows us to achieve a relative phase detuning approaching $\pi$ rad. This opens the possibility to use the injection-locked DFB semiconductor lasers for optical coherent PSK modulation.

\section{EXPERIMENT}

The employed experimental setup is shown in Fig. 1. Two identical DFB-BH laser diodes (Fujitsu FLD 150) having an emission wavelength of $1554 \mathrm{~nm}$ were used as master and slave. Both of them had one facet antireflection coated and the other facet cleaved. The master laser (ML) was biased at 2.5 times its threshold current with about $8 \mathrm{~mW}$ of optical output power, and the slave laser (SL) was biased at 1.75 times its threshold, which corresponds to $3 \mathrm{~mW}$ of optical output. Two diffraction limited lenses with 0.65 numerical aperture were used for beam coupling. Two optical isolators, inserted between ML and SL, provided more than $50 \mathrm{~dB}$ of isolation, and a $\lambda / 2$ plate was used to match the polarization of the two lasers. The coupled optical power was roughly evaluated from the photocurrent induced in the SL at zero bias. The maximum photocurrent obtained was about $150 \mu \mathrm{A}$ which, for a unitary quantum efficiency and a perfect matching of the laser mode, corresponds to about $120 \mu \mathrm{W}$. The variation of the injected optical power was obtained by a set of neutral density (ND) filters between ML and SL. Frequency matching and adjusting were accomplished by controlling the heat-sink temperature of the lasers. Both a 


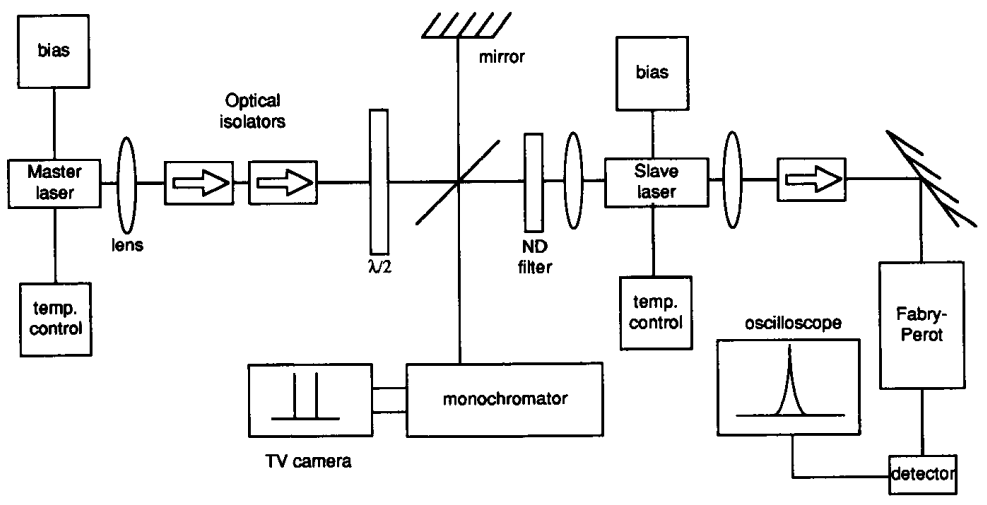

Fig. 1. Experimental setup.

monochromator and an FP scanning interferometer were used for measuring the spectrum of SL output. An isolator was also used to prevent optical feedback from the interferometer. The relative variation of the output power of the SL against the frequency detuning was indirectly measured through the electric voltage across the diode junction. The relative increase of the voltage is in fact directly proportional to that of the carrier density which is, in turn, proportional to the relative decrease of the photon density inside the laser cavity [2]. In this way, we were able to avoid the problem of field interference between the SL output and the directly transmitted or reflected light from the ML outside the SL's active waveguide.

When locking was achieved, the free running slave mode was quenched effectively, and the oscillation frequency of the SL was locked to that of the master laser. The measured stable locking range is given in Fig. 2, where locking is defined to be stable when the amplitude of either the spurious free running slave mode or the relaxation oscillation side bands are at least $20 \mathrm{~dB}$ below the locked main peak. This definition, in view of the applications to the practical coherent optical communications, seems more crucial and more relevant than that connected to the visibility of the interference fringes [2], [4], [5]. In this last case, in fact, a small amount of unlocked power or weak excitation of relaxation side bands is hard to be detected. In Fig. 2 and in the following, the power ratio $R$ is defined as the ratio between the externally injected optical power and that emitted from the SL. The two different detuning limits for stable locking are shown in the figure-one of them (open circles) determined by locking/unlocking, and the other one (points) by the onset of dynamic instability revealed by the raising of the relaxation oscillation side bands in the SL emission spectrum. We call them static and dynamic limits, respectively. Four different regimes of injection locking are experimentally well defined, as indicated in Fig. 2.

At low optical injection level (regime A), locking is unconditionally stable and no instability occurs. The most striking feature in this regime is that the stable locking range is symmetrically centered around the frequency of

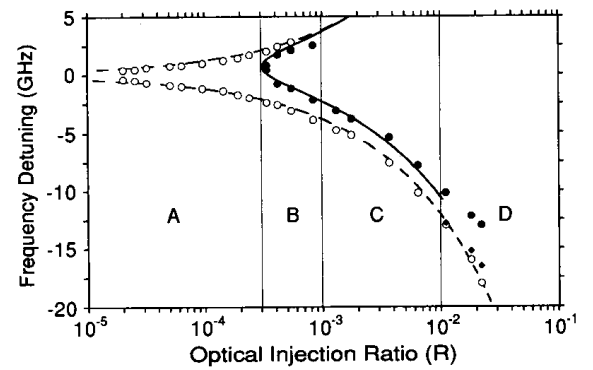

Fig. 2. Locking bandwidth against optical power ratio $R$. The circles refer to the measured dynamic limit, the points to the static limit, the diamonds to the onset of bistability. The curves represent the theoretical results: the solid line is the dynamic limit; the dotted line is the static limit.

the free-running SL. Contrary to the general behavior of FP lasers, in which the locked output power cannot become smaller than the free-running values [1]-[4], we found that the locked output power can increase for the negative detuning or decrease for positive detuning while neither relaxation-oscillation side bands nor free-running slave modes appear at all. A typical result of the measurements of the voltage variation across the diode junction and, hence, of the gain variation versus frequency detuning is given in Fig. 3(a).

In regime $B$, the stable locking range is split into two parts by an interval of instability. The amplitude of the locked main peak and the averaged amplitude of the two side peaks due to relaxation-oscillation (the two relaxation-oscillation sidebands usually have a different amplitude because of the phase-amplitude coupling [9]), measured by means of an FP interferometer for two different injection levels in regime B, are reported in Fig. 4. When the injection level increases, the range of positive detunings in which the stable locking is achieved gradually reduces and finally disappears. In regime $\mathrm{C}$, the system shows a typical asymmetric locking, which has been studied extensively in [1]-[4]. With very strong optical injection (regime D), bistability of locked output versus detuning is observed near the static boundary of the stable 


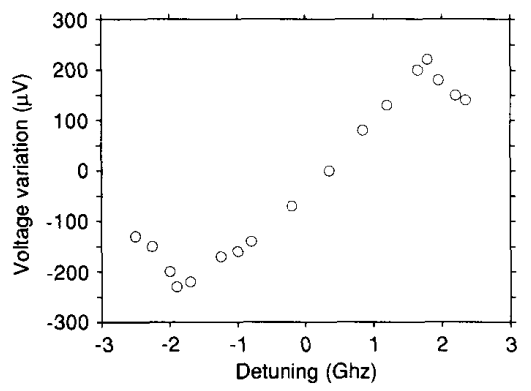

(a)

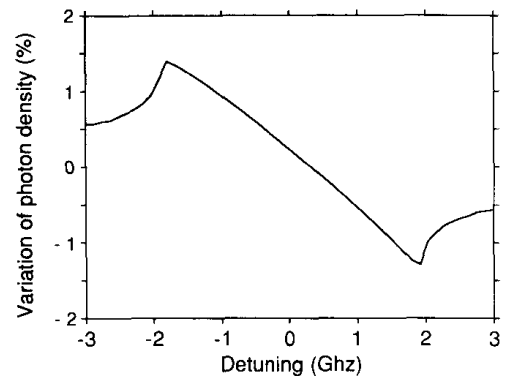

(b)

Fig. 3. (a) Experimental values of the junction voltage variations versus detuning; (b) relative photon density variation versus detuning obtained through a computer simulation.

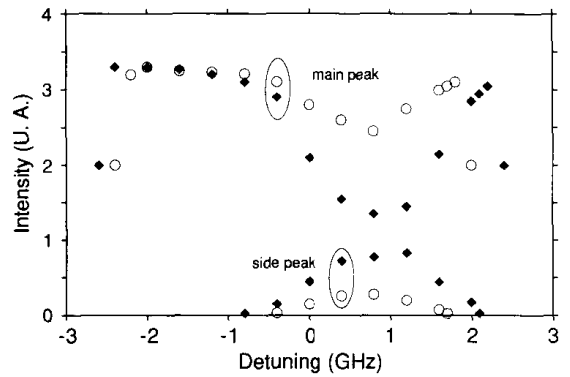

Fig. 4. Experimental values of the amplitude of the main peak and relaxation oscillation side peaks. Circles refer to $R=-36 \mathrm{~dB}$, diamonds to $R$ $=-34.5 \mathrm{~dB}$.

locking range, as shown in Fig. 5, where the stable locking bandwidth is reported for a value of $R$ equal to $3.5 \times$ $10^{-2}$. This kind of bistable behavior was first found by Kawaguchi et al. [7] when the SL was working very near to its threshold, and was found to disappear when the injection current was increased above 1.11 of its threshold. In our experiment it is found, however, that the most important parameter in determining the bistable behavior is the power ratio between the externally injected optical power and that emitted from the SL. Systematic measurements indicate that the width of the bistable frequency loop increases linearly with the logarithm of the injection optical power ratio as shown in Fig. 6. A bistable loop was found even for bias of the SL as high as two times of its threshold current.

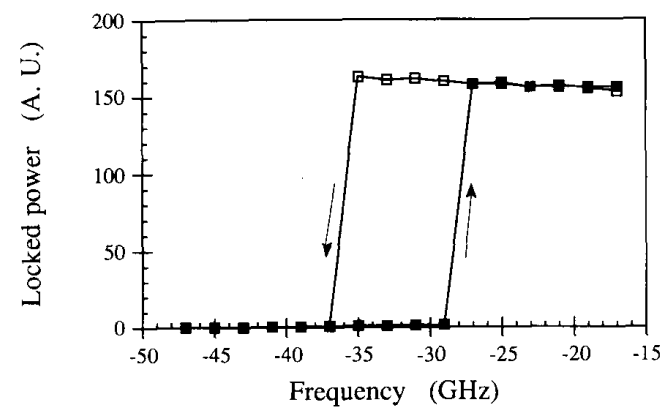

Fig. 5. Measured values of the locked power emitted by the SL versus frequency detuning for an optical power ratio $R=-14.6 \mathrm{~dB}$. Closed and open squares refer to the values obtained increasing and decreasing detuning, respectively.

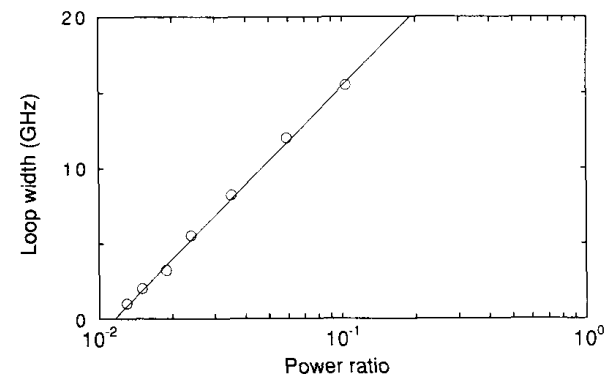

Fig. 6. Measured bistability loop width versus optical injection power ratio

\section{ANALYSIS}

In this section, the stability properties of the injection locking process will be studied on the basis of a theoretical model. In this model, we show the importance of the nonlinear gain saturation term to stabilize the injection locking.

In DFB semiconductor lasers, the side longitudinal modes are highly suppressed and the dynamic single mode operation is ensured by the mechanism of distributed feedback. Thus, it is reasonable to base our analysis on the well-known single mode Van der Pol equation and a rate equation for the carrier density [1]

$$
\begin{aligned}
\frac{d \beta(t)}{d t}= & {\left[-i \omega_{0}+\frac{1-i \alpha}{2} G_{N}\left(N-N_{0}\right)+\frac{1}{2} G_{I}|\beta(t)|^{2}\right] } \\
& \cdot \beta(t)+\frac{1}{\tau_{i}} \beta_{1}(t) \\
\frac{d N(t)}{d t}= & C(t)-\frac{N(t)}{\tau_{s}}-G|\beta(t)|^{2} .
\end{aligned}
$$

In these equations, $\beta(t)=I^{1 / 2} \exp \left[-i\left(\omega_{1} t-\phi\right)\right]$ is the normalized electric field of the SL inside the laser cavity, and $\beta_{1}(t)=I_{1}^{1 / 2} \exp \left[-i \omega_{1} t\right]$ is that coupled into the SL laser cavity from the ML; $I$ and $I_{1}$ are the field intensities of SL, and that coupled from ML; $G$ is the material gain; $G_{N}$ and $G_{I}$ are the derivatives of $G$ with respect to 
$N$ and $I ; \omega_{0}$ is the modal frequency of the slave cavity; $\omega_{1}$ is the frequency of ML; $N(t)$ is the carrier density; $N_{0}$ is the threshold carrier density; $C(t)$ is the carrier injection rate; $\phi$ represents the phase difference between SL and the $\mathrm{ML}$; and $\tau_{s}$ and $\tau_{i}$ are the spontaneous lifetime of minority carriers and the group round-trip time of SL, respectively. $G_{I}$ accounts for the nonlinear gain saturation effect. It should be mentioned here that the spatial hole-burning effect is omitted in the set of (1), and that our approach also neglects the noise due to the spontaneous emission events inside the SL cavity. The frequency and intensity noise in injection-locked semiconductor lasers has been extensively studied in [6].

In the stable locked state, the frequency of the SL is locked to that of the ML so that the stationary solutions are

$$
\begin{aligned}
\Delta G & =-2 \rho \cos \phi \\
\Delta \omega & =\rho(\sin \phi-\alpha \cos \phi)
\end{aligned}
$$

where $\Delta \omega=\omega_{1}-\omega_{0}$ is the frequency detuning; $\Delta G=$ $G_{N} \Delta N+G_{I} \Delta I, \Delta I$ and $\Delta N$ being the deviations of carrier density and photon density from their free running values; and $\rho=\left(I_{1} / I\right)^{1 / 2} / \tau_{i}$ is the normalized optical injection level.

The injection locking range is determined from ( $2 \mathrm{~b})$ by $|\Delta \omega| \leq \rho\left(1+\alpha^{2}\right)^{1 / 2}$. This sets the static limit of the locking range of Fig. 2, which corresponds to the range of the phase detuning from the value of $\tan ^{-1}(\alpha)-\pi / 2$ to $\tan ^{-1}(\alpha)+\pi / 2$.

All the points inside the two curves $\Delta \omega= \pm \rho(1+$ $\left.\alpha^{2}\right)^{1 / 2}$ are stationary solutions of the set of (1), but they are not always stable. Dynamic instability in injectionlocked semiconductor lasers has already been studied [2][4]. Only recently, however, the nonlinear gain saturation has been included in the evaluation of the dynamic stability range [10]. Physically, the nonlinear gain damps the relaxation-oscillation and tends to stabilize the single-frequency laser oscillation. By using the same method of [2][4], but including the nonlinear gain saturation, (1) can be linearized in terms of small deviations around the equilibrium values, namely, $I(t)=I+\delta I(t), \phi(t)=\phi+$ $\delta \phi(t), N(t)=N+\delta N(t), C(t)=C+\delta C(t)$. The following linearized equations can then be easily obtained:

$$
\begin{aligned}
\frac{d \delta I}{d t}= & \left(G_{I} I-\frac{\mathbf{R}}{I}-\rho \cos \phi\right) \delta I \\
& -2 \rho I \sin \phi \delta \phi+G_{N} I \delta N \\
\frac{d \delta \phi}{d t}= & \frac{\rho \sin \phi}{2 I} \delta I-\rho \cos \phi \delta \phi-\frac{\alpha G_{N}}{2} \delta N \\
\frac{d \delta N}{d t}= & -G \delta I-\left(G_{N} I+\frac{1}{\tau_{s}}\right) \delta N+\delta C
\end{aligned}
$$

where, for constant current injection, $\delta C=0$. The spontaneous emission rate $\mathbf{R}$ has been phenomenologically introduced into (3a); the same equation, however, can be obtained from (1a) when a proper Langevin noise term accounting for spontaneous emission noise is introduced [11].

Following the standard treatment as in [2] and [3], after the Fourier transformation and the Routh-Herwitz criterion were applied to (3), the damping time of the relaxation-oscillation can be obtained in the following simple form:

$$
\frac{1}{\tau_{R}}=\frac{1}{\tau_{S}}+\left(G_{N}-G_{I}\right) I+\frac{\mathbf{R}}{I}+\rho(\cos \phi-\alpha \sin \phi) .
$$

The stable locking is achieved when, after a slight perturbation of $\delta n, \delta \phi$, and $\delta I$, the system returns to its equilibrium state through a damped relaxation-oscillation. Quantitatively, this condition is verified when $\tau_{R}>0$. We define dynamic limit of the stable locking range the curve $\tau_{R}=0$. This curve is plotted in Fig. 2 by using the following laser parameters: $G_{N}=5.6 \times 10^{3} \mathrm{~s}^{-1}, G_{I}=$ $-1.8 \times 10^{4} \mathrm{~s}^{-1}, G=6.4 \times 10^{11} \mathrm{~s}^{-1}, 1 / \tau_{s}=3 \times 10^{9}$ $\mathrm{s}^{-1}, \alpha=6, \tau_{i}=8 \mathrm{ps}, \Omega_{R}=2 \pi * 6 \mathrm{GHz}$, where $\Omega_{R}=$ $\left(G G_{N} I\right)^{1 / 2}$. In order to compare the theoretical results to the experimental data, we assumed a homogeneous field in the cavity and an antireflection coating on the front facet of the SL so that $I_{1} / I$ is equal to $R$. In spite of the good agreement between the theoretically evaluated dynamic limit and the experimental data, one should notice that our theoretical and operative definitions of dynamical stability are different. The presence of spontaneous emission noise, in fact, increases the relaxation oscillation side bands beyond the $20 \mathrm{~dB}$ limit we set as the experimental threshold for the stability, even when $\tau_{R}$ is larger than zero.

By analyzing (4), one can observe that the term $-G_{I} I$ can be larger than both $G_{N} I$ and $1 / \tau_{s}$, so that $G_{I}$ is the main parameter affecting the stability properties, being the term $\mathbf{R} / I$ important only for very low values of $I$. Both $G_{l}$, which is always negative, and $\mathbf{R}$ increase the stable locking range of injection locking. This can explain why the stability analysis in [2], where both $G_{I}$ and $\mathbf{R}$ were not accounted for, gave results worse than those of the experimental measurements.

The use of (2) and (4) allows us to obtain the range of injection ratio where the laser is unconditionally stable within the whole locking band (regime A) and where the stability region is divided into two parts by an instability interval (regime B). The width of the regime A in Fig. 2 is obtained from (4) to be $0<\rho \leq 1 /\left[\tau_{R 0}\left(1+\alpha^{2}\right)^{1 / 2}\right]$. The width of regime $B$ can be calculated by searching for the cross point between static and dynamic limits, thus obtaining $1 /\left[\tau_{R 0}\left(1+\alpha^{2}\right)^{1 / 2}\right] \leq \rho \leq 1 /\left[\tau_{R 0}\left(1+\alpha^{2}\right)^{1 / 2}\right.$ $\left.\sin 2 \phi_{0}\right], \tau_{R 0}=\left[\left(G_{N}-G_{I}\right) I+\mathbf{R} / I+1 / \tau_{s}\right]^{-1}$ being the intrinsic damping time of the SL without optical injection, and $\phi_{0}=\tan ^{-1} \alpha$.

It is worth noting that the symmetry of the stable locking band in regime A [12], [13] and the existence of another stable locking range with positive detuning in regime B [12], have been experimentally observed only 
recently in DFB lasers, while they have not been found in FP lasers. In FP lasers, a symmetrical locking band was reported by Goldberg et al. [14]; but the definition of this band, i.e., the frequency distance at which the beating signal between ML and SL is reduced of $6 \mathrm{~dB}$ with respect to its maximum value, does not assure that this band is stable. Intuitively, one could believe that in DFB lasers, in which the side longitudinal modes are highly suppressed, the gain change expressed by (3a) makes the stable locking on the positive detuning range impossible, because the positive values of $\Delta G$ may induce the rising of the free-running main mode of SL. A computer simulation, carried out by integrating the set of (1) by means of a fourth-order Runge-Kutta method, however, shows that in regime $A$ the stable locking is always maintained in the static locking range, as experimentally obtained. The simulated photon density at steady state, in fact, is time independent within the static locking range, and becomes oscillatory outside this range, which corresponds to the case of four-wave mixing [15]. The time averaged relative photon density variation versus frequency detuning in regime $\mathrm{A}$ is reported in Fig. 3(b). The photon density increases, with respect to the free running SL, for negative frequency detunings, and decreases for positive detunings. Within the static locking range, the photon density variation, obtained by simulations, reproduces what is predicted by the steady-state solution of (1) and (2), i.e.,

$$
\frac{\Delta I}{I}=\frac{2 \rho \cos \phi}{G} \frac{1+\frac{1}{G_{N} I \tau_{s}}}{1-\frac{G_{I}}{G G_{N} \tau_{s}}} \approx \frac{2 \rho \cos \phi}{G}\left(1+\frac{1}{G_{N} I \tau_{s}}\right) .
$$

Out of the static locking band, however, the photon density gradually converges from both sides to the value pertaining to the free-running SL. In order to compare to the experimental measurement shown in Fig. 3(a), we recall that the relationship between the junction electric voltage variation $\Delta V$ and the photon density variation $\Delta i$ is $\Delta V=\mu \Delta N=-\mu\left(G+G_{I} I\right) \Delta I /\left(G_{N} I+1 / \tau_{s}\right)$, where $\mu$ is a constant determined by junction dependence and the external electric circuits, as described in [16]. A qualitative agreement between theory and experiment [Fig. 3(a)] is found. In FP semiconductor lasers, the symmetric locking range cannot be found because the gain margin between the main and the side longitudinal modes is relatively small. Hence, the gain decrease of the locked mode will result in multilongitudinal mode operation [3]; [4]. Moreover, the photon density decrease of the locked main mode in the positive detuning will be compensated by the contribution of the side longitudinal modes, and no output optical power decrease can be found [1], [3], [4].

\section{Discussion}

In this section, we give two examples of the application of the particular characteristics of the injection locked
DFB lasers which have been discussed in the previous sections. Both of them are based on the property of symmetric stable locking band at low injection levels.

\section{A. $\alpha$ Factor Measurement}

The linewidth enhancement factor $\alpha$ is a special and important parameter in semiconductor lasers. Many properties that are unique to semiconductor lasers can be traced back to this $\alpha$ parameter which, unfortunately, is not possible to be measured directly [8]. Up to date, the accuracy of the results of $\alpha$ measurement is not yet reliable. Light injection was proposed as a notable method to perform the measurement of the $\alpha$ parameter [1], [6]. In injection locked Fabry-Perot (FP) lasers, however, the multilongitudinal mode tendency prevents an easy measurement of the $\alpha$ parameter. Usually, the knowledge of the injected optical power is required, but this parameter is hard to precisely determine.

The symmetric stable locking band at low injection level in injection locked DFB lasers enables us to use a simple measurement technique for the $\alpha$ factor. Although based on the principle of optical injection locking, it does not require the knowledge of the absolute amount of the injection level [12].

From (3a) and (3b), the half-locking bandwidth can be found as $\Delta \omega_{m}=\rho\left(1+\alpha^{2}\right)^{1 / 2}$. On the other hand, the output optical power is equal to that of the free running SL, when $\phi=\pi / 2$, which corresponds to a detuning $\Delta \omega_{0}$ $=\rho$. The $\alpha$ value can then be easily determined by

$$
\alpha=\left[\left(\frac{\Delta \omega_{m}}{\Delta \omega_{0}}\right)^{2}-1\right]^{1 / 2}
$$

Experimentally, both values of $\Delta \omega_{m}$ and $\Delta \omega_{0}$ can be measured precisely if the stable injection locking is kept (regime A). It has to be noted that the $\alpha$ value measured with this technique is not the material linewidth enhancement factor, but it is the structure dependent effective one as defined in [17] and [18]. Several measurements of $\alpha$, performed at different injection levels within the regime $A$ of Fig. 2, using (6) are reported in Fig. 7. From this figure, $\alpha$ can be estimated to be $5.5 \pm 0.6$ in our case. This value agrees rather well with the value of 5 which can be obtained using the relationship $\Delta \omega_{m}=\rho\left(1+\alpha^{2}\right)^{1 / 2}$, although in this case the measurement suffers for the lack of precision in the evaluation of $\rho$.

\section{B. Optical PSK Modulation}

The optical PSK (DPSK) modulation scheme achieves the highest detection sensitivity in coherent optical communication systems [19]. Optical phase modulation using an injection-locked semiconductor laser was first proposed by Kobayashi et al. [20] in FP lasers. In that paper, a modulation of the current exciting the SL is shown to induce, besides intensity modulation, phase modulation instead of frequency modulation typical of free running lasers. Despite good agreement between theory and experiment, the effect of the linewidth enhancement factor 


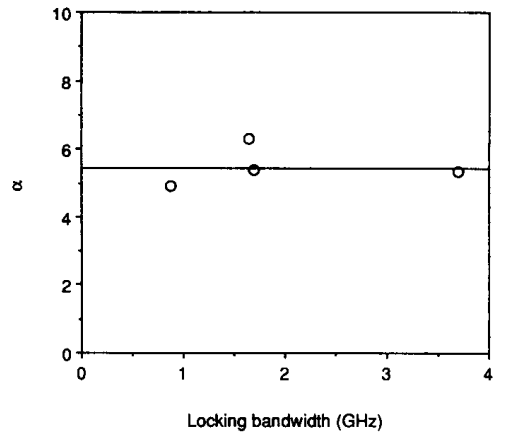

Fig. 7. Values of $\alpha$ measured at different optical injection levels.

$\alpha$ was neglected, and both instability and asymmetric locking band were not accounted for in this analysis.

The presence of a symmetric stable locking range in injection-locked DFB lasers opens the possibility of using these devices in optical PSK modulation. Although a theoretical model accounting for the $\alpha$ parameter is available in the case of small signal analysis [21], appropriate results for PSK modulation using injection-locked semiconductor lasers have not yet been found. In the following, we give the results of a small signal analysis obtained by means of a linearized theory and of numerical simulations accounting for the nonlinear effects, and predict some important effects limiting the range of applications of this modulation scheme.

The small signal phase modulation efficiency can be obtained after applying Fourier transformation to (3), as

$$
\left|\frac{\Phi(\omega)}{A(\omega)}\right|=\left|\frac{D_{1}}{D}\right|
$$

where

$$
\begin{aligned}
D_{1}= & \frac{1}{2} \Omega_{R}^{2}\left[\rho(\sin \phi-\alpha \cos \phi)+\alpha\left(G_{I} I-\frac{\mathbf{R}}{I}+i \omega\right)\right] \\
D= & \left(-i \omega-G_{I} I+\frac{\mathbf{R}}{I}+\rho \cos \phi\right)(-i \omega+\rho \cos \phi) \\
& \cdot\left(-i \omega+G_{N} I+\frac{1}{\tau_{s}}\right) \\
& +\Omega_{R}^{2}[\rho(\cos \phi+\alpha \sin \phi)-i \omega] \\
& +\rho^{2} \sin ^{2} \phi\left(-i \omega+G_{N} I+\frac{1}{\tau_{s}}\right)
\end{aligned}
$$

where $A(\omega)=\left[C(\omega) /\left(C-C_{t h}\right)\right], C(\omega)$ and $\Phi(\omega)$ are the Fourier transformations of $\phi(t)$ and $C(t)$, respectively, and $C_{t h}$ is the carrier injection rate at threshold.

The small signal phase modulation response given by (7) is reported in Fig. 8 for different levels of optical injection ratio. The values of frequency considered in this figure are in the range $0.1-10 \mathrm{GHz}$. The results shown cannot be extrapolated to lower frequencies because of thermal effects which are not taken into account in (7).

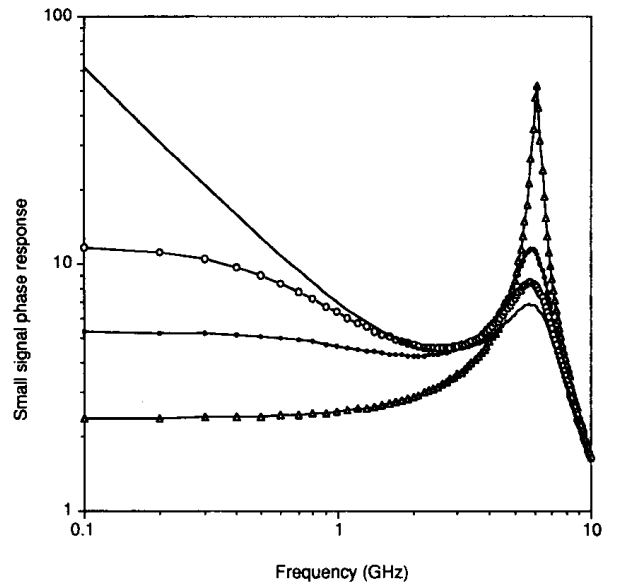

Fig. 8. Calculated small-signal phase modulation response versus modulation frequency at zero relative phase detuning for different optical injection levels. The solid line refers to free-running condition; circles to $R=$ $-44 \mathrm{~dB}$; points to $R=-41 \mathrm{~dB}$; triangles to $R=-37 \mathrm{~dB}$.

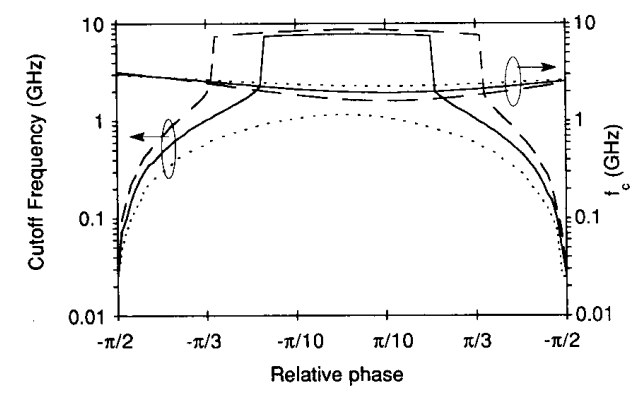

Fig. 9. Cutoff frequency and the relaxation-oscillation limit versus relative phase detuning for different optical injection levels, calculated in the small-signal approximation. The long-dotted line refers to $R=-44 \mathrm{~dB}$; the solid line to $R=-47 \mathrm{~dB}$; the short-dotted line to $R=-51 \mathrm{~dB}$.

Fig. 8 shows that the $3 \mathrm{~dB}$ modulation bandwidth slightly increases with the optical injection level, while the relaxation-oscillation peak becomes more pronounced, ending up in the unstable region. Another feature shown by (7) is the strong dependence of the modulation bandwidth on the phase detuning. This dependence is presented in Fig. 9 , where the $3 \mathrm{~dB}$ modulation bandwidth is reported versus the relative phase detuning, defined as $\psi=\phi-\tan ^{-1} \alpha$, for several different optical injection ratios. It is worth noting that a considerable decreasing of the modulation bandwidth is present when the relative phase detuning approaches $\pm \pi / 2$. This limits the dynamic range of phase modulation in practical use. In the same figure, the inverse of the damping time of the relaxation-oscillations $f_{c}$ $=\left(2 \pi \tau_{R}\right)^{-1}$, which represents the other limit for the modulation bandwidth, is also reported.

In practical PSK modulation systems, the relative phase detuning $\psi$ is usually shifted by a total amount equal to $\pi$ rad, so that the small signal analysis gives only a qualitative understanding of the dynamical behavior of the 
laser. To analyze the real case, computer simulations were carried out [22]. The results of simulations show that at high values of injection ratio, the modulation bandwidth is limited mainly by the relaxation oscillations. On the other hand, low injection ratios give rise to a high value of settling time, which further increases when the value of $\psi$ approaches $\pm \pi / 2$. By carefully adjusting the value of optical injection ratio $(R=-44 \mathrm{~dB})$, our simulations show that a turn-on (turn-off) settling time less than 1 ns can be obtained, when the minimum and maximum values of phase detuning $\psi$ are $\pm 0.42 \pi$.

\section{Conclusion}

In this paper, the external optical injection locking properties of DFB semiconductor lasers have been systematically studied. Due to the strong single longitudinal mode selection provided by the distributed grating, DFB lasers have some special properties with respect to FP lasers. These properties can be summarized as follows.

1) The presence of four regimes of operation: A symmetrical stable locking band at low injection ratio; two stable locking bands for positive and negative values of detuning separated by an unstable region at higher injection; stable locking only for negative values of detuning for still higher injection; and a bistable regime.

2) Stable injection locking which can be maintained also in regions in which the threshold gain is higher than that of the free-running SL. This regime cannot exist in FP lasers because of the relatively small gain margin between the main and the side longitudinal modes.

We have also shown that, even if the nonlinear gain has negligible effect on the static photon density variations, it strongly affects the dynamical properties of optical injection-locked semiconductor lasers.

Moreover, we have proposed a simple and accurate technique to measure the $\alpha$ factor in semiconductor lasers based on the symmetry of the locking band in DFB lasers. This method does not require the knowledge of the absolute moment of the injection level.

Finally, we have pointed out that injection-locked DFB semiconductor lasers can be used for optical PSK modulation. This possibility has been examined by using both small-signal analysis and large-signal numerical simulations.

\section{ACKNOWLEDGMENT}

The authors would like to thank A. Sapia and M. Tamburrini for useful discussions and suggestions.

\section{REFERENCES}

[1] C. H. Henry, N. A. Olsson, and N. K. Dutta, "Locking range and stability of injection locked $1.54 \mu \mathrm{m}$ InGaAsP semiconductor lasers," IEEE J. Quantum Electron., vol. QE-21, pp. 1152-1156, 1985.

[2] F. Mogensen, H. Olesen, and G. Jacobsen, "Locking conditions and stability properties for a semiconductor laser with external light injection," IEEE J. Quantum Electron., vol. QE-21, pp. 784-793. 1985.
[3] I. Petitbon, P. Gallion, G. Debarge, and C. Chabran, "Locking bandwidth and relaxation oscillation of an injection-locked semiconductor laser," IEEE J. Quantum Electron., vol. 24, pp. 148-154, 1988.

[4] R. Lang, "Injection locking properties of a semiconductor laser," IEEE J. Quantum Electron., vol. QE-18, pp. 976-983, 1982.

[5] S. Kobayashi and T. Kimura, "Injection locking in AlGaAs semiconductor lasers,"' IEEE J. Quantum Electron., vol. QE-17, pp. 681$689,1981$.

[6] P. Spano, S. Piazzolla, and M. Tamburrini, "Frequency and intensity noise in injection-locked semiconductor lasers: Theory and experiments," IEEE J. Quantum Electron., vol. QE-22, pp. 427-435, 1986.

[7] H. Kawaguchi, K. Inoue, and K. Otsuka, "Bistable output characteristics in semiconductor laser injection locking," IEEE J. Quantum Electron., vol. QE-21, pp. 1314-1317, 1985

[8] M. Osinski and J. Buus, "Linewidth broadening factor in semiconductor lasers-An overview," IEEE J. Quantum Electron., vol. QE-23, pp. 9-29, 1987.

[9] K. Vahala, C. H. Harder, and A. Yariv, "Observation of resonance effects in the field spectrum of semiconductor laser," Appl. Phys. Lett., vol. 21, pp. 211-213, 1983.

[10] O. Lidoyne, P. Gallion, C. Chabran, and G. Debarge, "Locking range, phase noise and power spectrum of an injection-locked semiconductor laser," IEE Proc., vol, 137, Pt. J, pp. 147-153, 1990.

[11] C. H. Henry, "Phase noise in semiconductor lasers," J. Lightwave Technol., vol. LT-4, pp. 298-311, 1986.

[12] R. Hui, A. Mecozzi, A. D'Ottavi, and P. Spano, "Novel measurement technique of a factor in DFB semiconductor lasers by injection locking,"' Electron. Lett., vol. 26, pp. 997-998, 1990.

[13] H. Nakajima, "Demodulation of mult1-gigahertz trequency-modulated optical signals in an injection-locked distributcu feedback laser oscillator," Electron. Lett., vol. 26, pp. 1129-1131, 1990.

[14] L. Goldberg, H. F. Taylor, and J. F. Weller, "Locking bandwidth asymmetry in injection locked GaAlAs lasers," Electron. Lett., vol. 18, pp. 986-987, 1982

[15] H. Nakajima and R. Frey, "Collinear nearly degenerate four-wave mixing in intracavity amplifying media," IEEE J. Quantum Electron., vol. QE-22, pp. 1349-1354, 1986

[16] D. Marcuse, "Heterodyne detection with an injection laser-Part I: Principle of operation and conversion efficiency," IEEE J. Quantum Electron., vol. 26, pp. 85-93, 1990.

[17] G. Duan, P. Gallion, and G. Debarge, "Analysis of the phase-amplitude coupling factor and spectral linewidth of distributed feedback and composite cavity semiconductor lasers," IEEEJ. Quantum Electron., vol. 26, pp. 32-44, 1990.

[18] B. Tromborg, H. Olesen, X. Pan, and S. Saito, "Transmission line description of optical feedback and injection locking for Fabry-Peror and DFB lasers," IEEE J. Quantum Electron., vol. QE-23, pp. 1875 1889,1987

[19] T. Kimura, "Coherent optical fiber transmission," J. Lightwave Technol., vol. LT-5, pp. 414-428, 1987.

[20] S. Kobayashi and T. Kimura, "Optical phase modulation in an injection locked AlGaAs semiconductor laser," IEEE Trans. Microwave Theory Tech., vol. MTT-30, pp. 1650-1657, 1982.

[21] S. Piazzolla, P. Spano, and M. Tamburrini, "Small signal analysis of frequency chirping in injection-locked semiconductor lasers," IEEE J. Quantum Electron., vol. QE-22, pp. 2219-2223, 1986.

[22] R. Hui, "Optical PSK modulations using injection locked DFB semiconductor lasers,"' IEEE Photon. Technol. Lett., Oct. 1990.

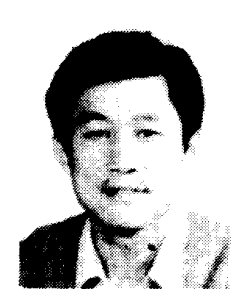

Rongqing Hui was born in Anhui, China, on June 30,1957 . He received the B. Sc. degree in microwave communication and the M.Sc. degree in lightwave technology, both from Beijing University of Posts and Telecommunications, Beijing, China, in 1982 and 1987, respectively.

From 1982 to 1985 he taught at the Department of Physics, Anhui University, Anhui, China, where his research interest was in optical fiber sensors. From 1985 to 1989 he worked in the Department of Electronics Engineering, Beijing University of Posts and Telecommunications, where he conducted research into coherent optical transmission systems, single-mode single-polarized optical fibers, and semiconductor lasers with optical feedback. During 1989 
and 1990 he held a research fellowship at the Fondazione Ugo Bordoni Rome, Italy, where his research interests were optical bistability, fourwave mixing, and optical injection locking of semiconductor lasers. He is now with the Electronics Department, Politecnico di Torino, Torino, Italy. He also holds a research fellowship from the Italian Telecommunication Research Center (CSELT), Torino, Italy. His main research interests are semiconductor laser devices and coherent optical transmission systems.

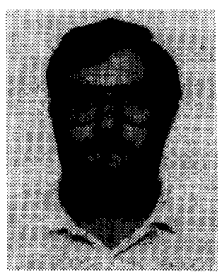

Alessandro D'Ottavi was born in Rome, Italy, on January 29,1962 . He studied physics at the University of Rome and received the degree in 1988 . $\mathrm{He}$ is currently working in the field of semiconductor lasers at the Fondazione Ugo Bordoni.
Antonio Mecozzi, for a photograph and biography, see p. 343 of the March 1991 issue of this JOURNAL.
Paolo Spano, for a photograph and biography, see p. 343 of the March 1991 issue of this Journal. 